Copyright (C) 2006 IEEE. Reprinted from IEEE Transactions on Communications, 2006; 54 (2):221-225

This material is posted here with permission of the IEEE. Such permission of the IEEE does not in any way imply IEEE endorsement of any of the University of Adelaide's products or services. Internal or personal use of this material is permitted. However, permission to reprint/republish this material for advertising or promotional purposes or for creating new collective works for resale or redistribution must be obtained from the IEEE by writing to pubs-permissions@ieee.org.

By choosing to view this document, you agree to all provisions of the copyright laws protecting it. 


\title{
Carrier-Phase and Frequency-Estimation Bounds for Transmissions With Embedded Reference Symbols
}

\author{
Feng Rice
}

\begin{abstract}
The true Cramér-Rao lower bound (CRLB) for the joint estimation of carrier phase and frequency is derived for transmission bursts with interleaved reference and phase-shift keying/quadrature amplitude modulated data symbols. Results are presented for the special cases of midamble, and preamble and postamble, pilot-symbol insertion. The derivation reveals that the CRLB is a function of the location of the reference symbols in the burst, the number of reference symbols, the number of data symbols, the signal-to-noise ratio and the data-modulation scheme. By distributing the reference symbols symmetrically about the center of the burst and analyzing relative to the middle of the signal vector, the joint frequency and phase estimation can be decoupled, and the optimal phase estimation is achieved. In the decoupled case, the phase CRLB is independent of the location of reference symbols in the burst. In a symmetrical burst, the use of a preamble and postamble is found to provide a lower frequency-estimation CRLB than that with a midamble. It appears that the frequency CRLB is reduced as the reference symbols are symmetrically distributed closer to the ends of the burst.
\end{abstract}

Index Terms-Cramér-Rao lower bounds (CRLBs), frequency estimation, phase estimation, synchronization.

\section{INTRODUCTION}

$\mathbf{T}$ HE true Cramér-Rao lower bounds (CRLBs) for joint frequency and phase estimation from unknown data symbols (blind operation) of modulated signals have been presented in [1]-[4]. The CRLBs for modulated signals can be expressed as the CRLBs of a carrier wave (CW) divided by a function of modulation and noise power, i.e., $\mathrm{CRLB}_{\text {modulated }}=\mathrm{CRLB}_{\mathrm{cw}} / F\left(\sigma^{2}\right)$, assuming that the $\mathrm{CW}$ and the modulated signal have average unity power. The $F\left(\sigma^{2}\right)$ is a function of the geometry of the signal set and the energy-per-symbol-to-noise-density ratio $E_{s} / N_{0}$. This applies to both phase and frequency estimation [1].

In certain scenarios, such as packet transmission for timedivision multiple-access (TDMA) systems, the data symbols alone may be insufficient for effective synchronization of the burst. This has motivated the use of synchronization overhead in the form of inserted reference symbols. These are sometimes grouped into a preamble, postamble, or midamble, or alternatively distributed throughout the packet as pilots. In the most general case, the reference symbols are arbitrarily interleaved

Paper approved by R. De Gaudenzi, the Editor for Synchronization and CDMA of the IEEE Communications Society. Manuscript received July 10, 2003; revised January 27, 2004; March 5, 2005; July 27, 2005; and August 27, 2005. This work was supported by the Cooperative Research Center for Sensor Signal and Information Processing.

The author was with the Department of Electrical and Electronic Engineering, University of Adelaide, Adelaide, SA 5005 Australia. She is now with Saab Systems, Mawson Lakes, SA 5095 Adelaide, Australia (e-mail: feng.rice@ saabsystems.com.au).

Digital Object Identifier 10.1109/TCOMM.2005.863782 with data symbols in some predetermined order. Given that reference symbols are inserted, the synchronization performance may be enhanced where both data and reference symbols are used to estimate the received signal parameters. Previous CRLB results may be used to approximate the best-case estimator performance with either known symbols at low signal-to-noise ratio (SNR) or unknown data symbols at high SNR. It is known that estimator performance may be enhanced by combining algorithms for both known and unknown data, especially at practical operating SNRs. However, the CRLB for these cases has not been well understood. This letter derives the CRLB for an arbitrary mixture of interleaved reference and data symbols. The letter investigates the synchronizer CRLBs for various design-burst structures, such as number of the reference symbols and their location in the burst. A static additive white Gaussian noise (AWGN) channel is assumed.

Section II derives the CRLBs for joint frequency and phase estimation with the interleaved data and reference burst. Since the location of the reference symbols is important for the frequency CRLB, various frame structures are investigated. It is found that a symmetrical burst can decouple the joint frequency and phase estimation, which means that the frequency-estimation error has no impact on the phase estimate. Therefore, the optimal phase estimation is obtained.

Section III investigates the effect of the location of the reference symbols on the frequency-estimation accuracy. It is shown that the performance of the symmetrical burst with preamble and postamble is superior to that of the symmetrical burst with midamble at low and moderate SNR. Brief conclusions are presented in Section IV.

\section{CRLBS FOR JoINT FREQUENCY AND PhASE Estimation}

Fig. 1 illustrates a general reference and data-interleaved transmission burst. The length of the burst is $N$ symbols, $D$ is the total number of data symbols, $D_{i}$ is the number of data symbols in the $i$ th subgroups, $L$ is the total number of reference symbols in the burst, $L_{i}$ is the number of reference symbols in the $i$ th subgroups, $n$ is the number of subgroups of reference symbols, and $m$ is the number of the subgroups of data symbols.

We initially assume that the received signal has perfect symbol timing, and the frequency offset is relatively small so that the received signal is free of intersymbol interference. The received signal is given by

$$
x_{k}=a_{k} e^{j(k \omega+\phi)}+w_{k}, \quad k=\left\{\begin{array}{c}
0,1 \ldots, N-1 \text { or } \\
-\frac{N-1}{2}, \ldots, \frac{N-1}{2}
\end{array}\right.
$$

where $\omega$ is the frequency offset in radians per symbol period, $\phi$ is a fixed, arbitrary value, and $a_{k}$ are the normalized transmitted data symbols belonging to an arbitrary 


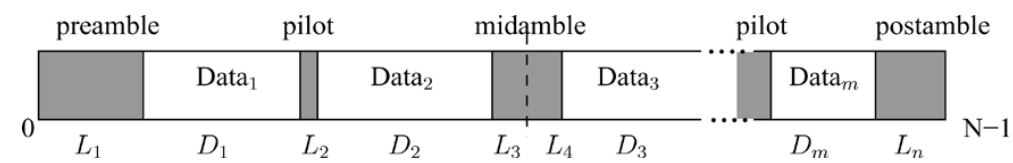

Fig. 1. Reference and data-interleaved transmission burst.

phase-shift keying (PSK)/quadrature amplitude modulation (QAM) constellation. For example, with square 16QAM $\mathbf{C}=(1 / \sqrt{10})\{ \pm 1 \pm j, \pm 3 \pm 3 j, \pm 3 \pm j, \pm 1 \pm 3 j\}$. The average power of the reference symbols is $E_{p}=(1 / L) \sum_{k=1}^{L}\left|a_{k}\right|^{2}$. $w_{k}$ is the $k$ th noise sample, whose real and imaginary parts are independent zero-mean Gaussian random variables, each with variance $\sigma^{2}$, and the $w_{k}$ 's are mutually independent. The symbol SNR is $E_{s} / N_{0}=1 /\left(2 \sigma^{2}\right)$. The estimator is defined at index $k=0$. For the case where the estimator is derived at the center of the burst, $k \in \mathcal{K}_{S}=[-(N-1) / 2,(N-1) / 2]$, and we define this as $\mathcal{K}_{S}$. If the estimator is derived at the start of the burst, $k \in \mathcal{K}_{N}=[0, N-1]$, we define it as $\mathcal{K}_{N}$. The symmetrical burst $\mathcal{K}_{S}$ can decouple the joint estimation, and it will be discussed later.

We use the notation $p(x)$ for the probability density function (pdf) of the whole interleaved signal vector, $p_{\text {dat }}(x)$ for the pdf for the data part, and $p_{\text {ref }}(x)$ for the pdf of the reference part. Boldface symbols denote vectors. The pdf of the received signal vector $\mathbf{x}$ is given by

$$
\begin{aligned}
& p(\mathbf{x} \mid \omega, \phi) \\
& =p\left(x_{0} \mid \omega, \phi\right) p\left(x_{1} \mid \omega, \phi\right) p\left(x_{2} \mid \omega, \phi\right) \ldots p\left(x_{N-1} \mid \omega, \phi\right) \\
& =\prod_{k=0}^{L_{1}-1} p_{\text {ref }}\left(x_{k} \mid \omega, \phi\right) \prod_{k=L_{1}}^{L_{1}+D_{1}-1} p_{\text {dat }}\left(x_{k} \mid \omega, \phi\right) \\
& \quad \times \prod_{k=L_{1}+D_{1}}^{L_{1}+D_{1}+L_{2}-1} p_{\text {ref }}\left(x_{k} \mid \omega, \phi\right) \prod_{k=L_{1}+D_{1}+L_{2}}^{L_{1}+D_{1}+L_{2}+D_{2}-1} p_{\text {dat }}\left(x_{k} \mid \omega, \phi\right) \\
& \times \cdots \times \prod_{k=\left(\sum_{i=1}^{n} L_{i}+\sum_{i=1}^{m} D_{i}\right)}^{p_{\text {ref }}\left(x_{k} \mid \omega, \phi\right) .}
\end{aligned}
$$

For a single received reference symbol, we have

$$
\begin{aligned}
p_{\text {ref }}\left(x_{k} \mid \omega, \phi\right) & =\frac{1}{2 \pi \sigma^{2}} e^{-\left(1 / 2 \sigma^{2}\right)\left|x_{k}-a_{k} e^{j(k \omega+\phi)}\right|^{2}} \\
& =\frac{1}{2 \pi \sigma^{2}} e^{-\left(\left|x_{k}\right|^{2}+\left|a_{k}\right|^{2}\right) / 2 \sigma^{2}} e^{\left(1 / \sigma^{2}\right) \Re\left(x_{k} a_{k}^{*} e^{-j(k \omega+\phi)}\right)}
\end{aligned}
$$

where $a_{k}$ is known symbol, $a^{*}$ indicates the complex conjugate, and $\Re$ and $\Im$ stand for real and imaginary parts.

For a single received data symbol, we have

$$
\begin{aligned}
p_{\text {dat }}\left(x_{k} \mid \omega, \phi\right)= & p_{A}(a) \frac{1}{2 \pi \sigma^{2}} e^{-\left|x_{k}\right|^{2} / 2 \sigma^{2}} \\
& \cdot \sum_{a_{i} \in C} e^{-\left|a_{i}\right|^{2} / 2 \sigma^{2}} e^{\left(1 / \sigma^{2}\right) \Re\left(x_{k} a_{i}^{*} e^{-j(\omega+\phi)}\right)}
\end{aligned}
$$

where the sumation is taken over all possible constellation points $a_{i} \in \mathbf{C}$. We assume that the transmitted symbols are equally likely, i.e., $p_{A}\left(a_{i}\right)$ is independent of $i$.

Inserting (3) and (4) into (2), and then taking the logarithm and retaining the terms depending on $\phi$, we obtain the corresponding log-likelihood function

$$
\begin{aligned}
\ln p(\mathbf{x} \mid \omega, \phi) & \sum_{k=0}^{L_{1}-1} \frac{1}{\sigma^{2}} \Re\left(x_{k} a_{k}^{*} e^{-j(k \omega+\phi)}\right) \\
= & +\sum_{k=L_{1}}^{L_{1}+D_{1}-1} \ln \sum_{a_{i} \in C} e^{-\left|a_{i}\right|^{2} / 2 \sigma^{2}} e^{\left(1 / \sigma^{2}\right) \Re\left(x_{k} a_{i}^{*} e^{-j(\omega+\phi)}\right)} \\
& +\sum_{k=L_{1}+D_{1}}^{L_{1}+D_{1}+L_{2}-1} \frac{1}{\sigma^{2}} \Re\left(x_{k} a_{k}^{*} e^{-j(\omega+\phi)}\right) \\
& +\sum_{k=L_{1}+D_{1}+L_{2}}^{L_{1}+D_{1}+L_{2}+D_{2}-1} \\
& \cdot\left[\ln \sum_{a_{i} \in C} e^{-\left|a_{i}\right|^{2} / 2 \sigma^{2}} e^{\left(1 / \sigma^{2}\right)} \Re\left(x_{k} a_{i}^{*} e^{-j(\omega+\phi)}\right)\right] \\
& +\ldots+\sum_{k=\left(\sum_{i=0}^{n-1} L_{i}+\sum_{i=1}^{m} D_{i}\right)}^{\frac{1}{\sigma^{2}} \Re\left(x_{k} a_{k}^{*} e^{-j(\omega+\phi)}\right) .}
\end{aligned}
$$

The Fisher information matrix (FIM) is given by [5]

$$
\begin{aligned}
\mathbf{I}= & \left(\begin{array}{cc}
-E\left[\frac{\partial^{2} \ln p(\mathbf{x} \mid \omega, \phi)}{\partial \omega^{2}}\right] & -E\left[\frac{\partial^{2} \ln p(\mathbf{x} \mid \omega, \phi)}{\partial \omega \partial \phi}\right] \\
-E\left[\frac{\partial^{2} \ln p(\mathbf{x} \mid \omega, \phi)}{\partial \omega \partial \phi}\right] & -E\left[\frac{\partial^{2} \ln p(\mathbf{x} \mid \omega, \phi)}{\partial \phi^{2}}\right]
\end{array}\right) \\
= & \frac{E_{p}}{\sigma^{2}}\left(\begin{array}{cc}
\sum_{k \in k_{\text {ref }}} k^{2}\left|a_{k}\right|^{2} & \sum_{k \in k_{\text {ref }}} k\left|a_{k}\right|^{2} \\
\sum_{k \in k_{\text {ref }}} k\left|a_{k}\right|^{2} & \sum_{k \in k_{\text {ref }}}\left|a_{k}\right|^{2}
\end{array}\right) \\
& +\frac{F\left(\sigma^{2}\right)}{\sigma^{2}}\left(\begin{array}{cc}
\sum_{k \in k_{\text {dat }}} k^{2} & \sum_{k \in k_{\text {dat }}} k \\
\sum_{k \in k_{\text {dat }}} k & \sum_{k \in k_{\text {dat }}} 1
\end{array}\right)
\end{aligned}
$$

where the first matrix is related to the reference symbols and the second matrix is related to the data symbols, $\mathrm{E}[\cdot]$ denotes statistical expectation with respect to the pdf $p(\mathbf{x} \mid \omega, \phi)$. The modulation function $F\left(\sigma^{2}\right)$ is the ratio of the CW CRLB to the CRLB of the modulated signal [1].

To simplify the notation, the shorthand definition of the FIM is

$$
\boldsymbol{I}=\left(\begin{array}{ll}
I_{\omega \omega} & I_{\omega \phi} \\
I_{\omega \phi} & I_{\phi \phi}
\end{array}\right)
$$




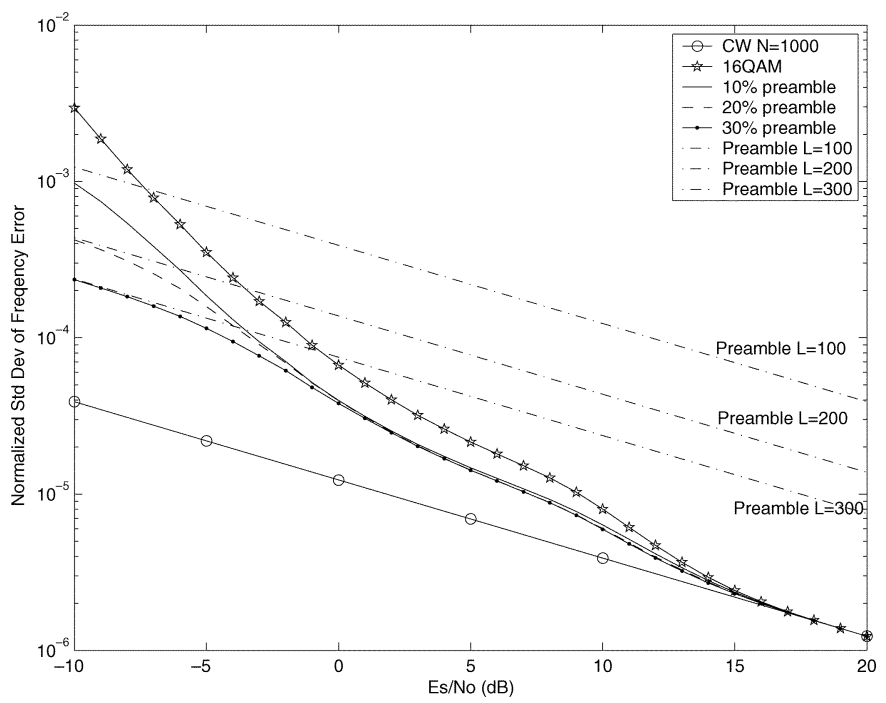

Fig. 2. Frequency CRLBs for preamble-assisted frame structure, $N=1000$, 16QAM with various $L$.

For example, the frame structure is preamble, followed by one block of information data symbols. The range of $k$ is $[0,1, \ldots, N-1]$. The corresponding elements of the FIM from (6) are

$$
\begin{aligned}
I_{\omega \phi}= & \frac{2 E_{s}}{N_{0}}\left[\frac{E_{p} L(L-1)}{2}+F\left(\sigma^{2}\right)\left(\frac{D(D-1)}{2}+L D\right)\right] \\
I_{\omega \omega}= & \frac{2 E_{s}}{N_{0}}\left[\frac{E_{p} L(L-1)(2 L-1)}{6}\right. \\
& \left.+F\left(\sigma^{2}\right)\left(\frac{D(D-1)(2 D-1)}{6}+D(D-1) L+D L^{2}\right)\right] \\
I_{\phi \phi}= & \frac{2 E_{s}}{N_{0}}\left[E_{p} L+F\left(\sigma^{2}\right) D\right] .
\end{aligned}
$$

Fig. 2 shows the CRLBs for frequency estimation. The data symbols are 16QAM and the burst length $N=1000$. The solid, dashed, and dot-solid lines represent $10 \%, 20 \%$, and $30 \%$ preamble $\left(E_{p}=1\right)$ in the burst, respectively. The circle line "o-" is the CW CRLB (100\% reference symbols), and the star line is the CRLB for a burst with 100\% 16QAM data symbols. The CRLBs with preamble-assisted bursts are bounded between these two lines. At high SNR, the CRLBs of the preamble-assisted burst converge to the CW CRLB. At low SNR, the reference symbols dominate the estimation, and the burst CRLBs asymptotically approach the preamble CRLBs, which agrees with [6, eq. (36)].

Note that the elements $I_{\omega \omega}$ and $I_{\omega \phi}$ depend on the range of $k$ and the location of the reference symbols. In (6), the core of element $I_{\omega \phi}$ is given by $\sum_{k} k$. If $k$ is selected so that the sampling times are symmetrically located about zero, and also the reference symbols are distributed symmetrically about the center of the burst, then we have

$$
\sum_{-(N-1) / 2}^{(N-1) / 2} k=0
$$

where $N$ is an odd number.

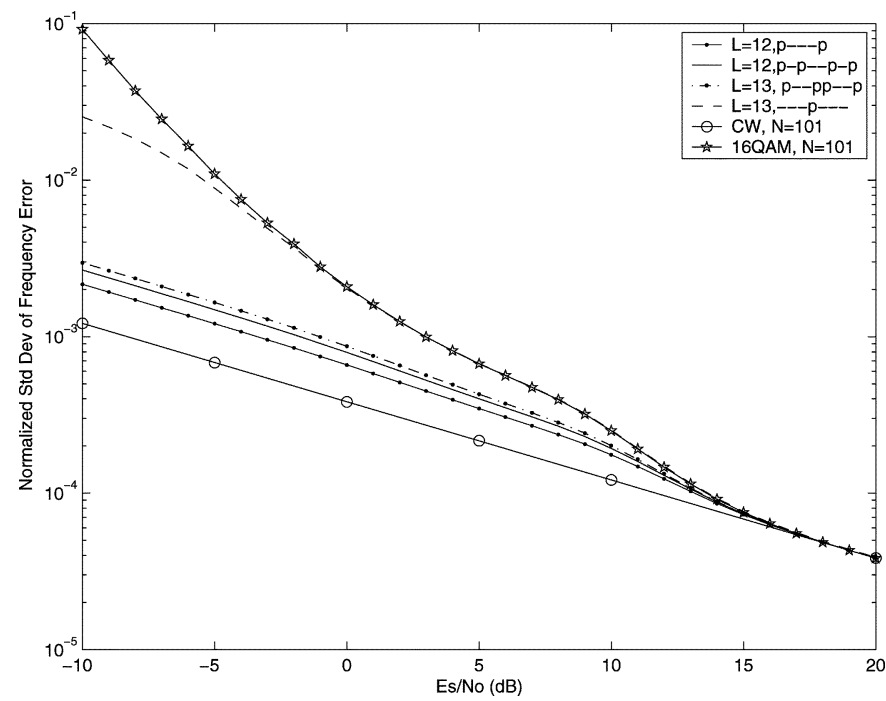

Fig. 3. Comparison of the CRLBs for MTB and DCTB, $N=101$, 16QAM modulation.

Under this condition, the FIM is a diagonal matrix, which means that the joint frequency and phase estimation are decoupled. The frequency-estimate error will not affect the phase estimation. The frequency CRLB will be obtained in the next section. The decoupled phase CRLB is given by

$$
\mathrm{CRLB}^{-1}(\hat{\phi})=2 \frac{E_{s}}{N_{0}}\left[E_{p} L+F\left(\sigma^{2}\right) D\right]
$$

\section{EFFECT OF THE LOCATION OF REFERENCE SYMBOLS ON FREQUENCY ACCURACY}

In the following, we restrict our attention to symmetrical burst structures. Still, there are many possible arrangements of data and reference symbols. In general, we can divide them into two categories: 1) bursts with a reference symbols placed in the middle of the burst are called midamble transmission bursts (MTB); 2) bursts with a data symbol placed in the middle of the burst are called data-centered transmission bursts (DCTB). The remaining reference symbols are symmetrically placed about the center of the burst.

Fig. 3 shows the comparison of the CRLBs for MTB and DCTB. The transmission bursts are of the same length, and contain $L=13$ for MTB and $L=12$ for DCTB (six reference symbols at each end of the burst). The symbol "p-" is a shorthand notation for a burst structure, "_-" stands for data symbols, and "p" for preamble/pilot/postamble/midamble symbols. The legend symbols of Fig. 3 "p-p" and "p-p-p-p" are for DCTB with two and four subgroups of reference symbols, respectively. It is interesting to observe that the frequency CRLBs of DCTB are lower than that of the "-pp-" MTB, although MTB has one extra known symbol. Moreover, if we move six of the symbols of the "-p-" MTB to the two ends of the burst forming "p-pp-p," which is three reference symbols at each end and $7(2 \times 3+1)$ reference symbols in the middle of the burst, the frequency CRLB (standard deviation) decreases by a factor of 10 at low SNR. This result shows that the distribution of the the preamble and 
postamble plays a significant role in frequency estimation. It appears that the frequency CRLB is reduced as the reference symbols are located closer to the ends of the burst.

The phase CRLBs for MTB or DCTB are the same since, the decoupled phase CRLB is independent of the location of the reference symbols. (Of course, the phase CRLB of $L=13$ is slightly lower than that of $L=12$.)

The generalized frequency CRLB equations for DCTB and MTB are given by (8), shown at the bottom of the page, where $L$ and $n$ are even numbers, and $D$ is an odd number, i.e., the reference symbols are arranged into $n$ groups of $L / n$, and located at intervals of integer $\lfloor(D-1) / n\rfloor$ data symbols from the central data symbol. We then have (9), shown at the bottom of the page, where $L$ and $N$ are odd numbers, $n$ and $D$ are even, and $n \geq 2$, i.e, there are $2((L-1) / n)+1$ in the center of the burst.

Note that the terms within the first curly brackets in (8) and (9) are the reference-symbol contribution to the CRLB, and the terms within the second curly brackets are the data-symbol contribution to the CRLB. As SNR decreases, $F\left(\sigma^{2}\right)$ becomes small [1]. At low SNR, the second curly brackets are small enough that they can be ignored (intuitively, the data symbols are less significant to the estimator at low SNR).

Fig. 4 shows the CRLBs for the DCTB with various reference-symbol distributions. It reveals that the distribution with reference symbols located at the two ends of the burst $(n=2)$ obtains the lowest CRLB, compared with other numbers of subgroup $n$ at given $L$. As $n$ becomes large, the frequency-estimation error increases. The worst case is the one symbol per pilot group uniformly distributed in the burst. The reason is that the frequency CRLB is sensitive to the length of the subgroup $L / n$ and $D / n$. At low SNR, as $n$ decreases, the term $(L / n)^{3}$

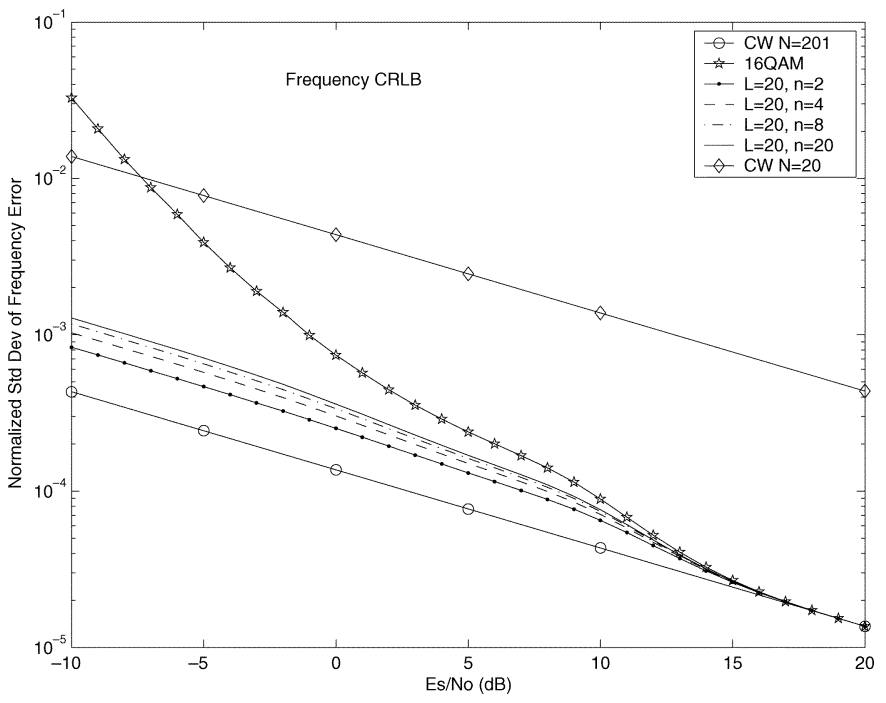

Fig. 4. Comparison of CRLBs for various DCTB 16QAM with $L=20, N=$ 201.

increases faster than the lower order terms (term $(D / n)^{3}$ scaled down by $F\left(\sigma^{2}\right)$, where $\left.0<F\left(\sigma^{2}\right) \leq 1\right)$.

\section{CONCLUSION}

New carrier-phase and frequency CRLBs for transmissions with embedded reference symbols have been derived. Since the location of the reference symbols is important for the CRLBs, various burst structures have been investigated. In the case of a symmetrical burst $\mathcal{K}_{S}$, the joint frequency and phase estimators can be decoupled, and the optimal phase estimate is achieved.

$$
\begin{aligned}
\operatorname{CRLB}_{\mathrm{DCTB}}^{-1}(\hat{\omega})= & \frac{2 E_{s} E_{p}}{N_{0}}\left\{n \frac{\frac{L}{n}\left(\frac{L}{n}-1\right)\left(\frac{2 L}{n}-1\right)}{6}-\frac{n}{2} \frac{L}{n}\left(\frac{L}{n}-1\right)^{2}\right. \\
& \left.+\frac{L}{n} \sum_{i=1}^{n / 2}\left[\left(-\frac{N-1}{2}+(i-1) \frac{L+D-1}{n}\right)^{2}+\left(\frac{N-1}{2}-\frac{i L}{n}-(i-1) \frac{D-1}{n}+1\right)^{2}\right]\right\} \\
& +\frac{2 E_{s}}{N_{0}} F\left(\sigma^{2}\right)\left\{n \frac{\frac{D-1}{n}\left(\frac{D-1}{n}-1\right)\left(\frac{2 D-2}{n}-1\right)}{6}-\frac{n}{2} \frac{D-1}{n}\left(\frac{D-1}{n}-1\right)^{2}\right. \\
& \left.+\frac{D-1}{n} \sum_{i=1}^{n / 2}\left[\left(-\frac{N-1}{2}+i \frac{L}{n}+(i-1) \frac{D-1}{n}\right)^{2}+\left(\frac{N-1}{2}-i \frac{L+D-1}{n}+1\right)^{2}\right]\right\} \\
\mathrm{CRLB}_{\mathrm{MTB}}^{-1}(\hat{\omega})= & \frac{2 E_{s} E_{p}}{N_{0}}\left\{n \frac{\frac{L-1}{n}\left(\frac{L-1}{n}-1\right)\left(\frac{2 L-2}{n}-1\right)}{6}-\frac{n}{2} \frac{L-1}{n}\left(\frac{L-1}{n}-1\right)^{2}\right. \\
& \left.+\frac{L-1}{n} \sum_{i=1}^{n / 2}\left[\left(-\frac{N-1}{2}+i \frac{D}{n}+(i-1) \frac{L-1}{n}\right)^{2}+\left(\frac{N-1}{2}-i \frac{D+L-1}{n}+1\right)^{2}\right]\right\} \\
& +\frac{2 E_{s}}{N_{0}} F\left(\sigma^{2}\right)\left\{n \frac{\frac{D}{n}\left(\frac{D}{n}-1\right)\left(\frac{2 D}{n}-1\right)}{6}-\frac{n}{2} \frac{D}{n}\left(\frac{D}{n}-1\right)^{2}\right. \\
& \left.+\frac{D}{n} \sum_{i=1}^{n / 2}\left[\left(-\frac{N-1}{2}+(i-1) \frac{D+L-1}{n}\right)^{2}+\left(\frac{N-1}{2}-\frac{i D}{n}-(i-1) \frac{L-1}{n}+1\right)^{2}\right]\right\}
\end{aligned}
$$


Furthermore, the symmetrical burst with preamble and postamble achieves lower frequency estimation variance than the burst with midamble. It appears that frequency CRLB is reduced as the reference symbols are located closer to the ends of the burst.

\section{ACKNOWLEDGMENT}

The author would like to acknowledge helpful discussions with Dr. M. Rice that motivated the work.

\section{REFERENCES}

[1] F. Rice, B. Cowley, B. Moran, and M. Rice, "Cramér-Rao lower bounds for QAM phase and frequency estimation," IEEE Trans. Commun., vol. 49, no. 9, pp. 1582-1591, Sep. 2001.

[2] F. Rice, M. Rice, and B. Cowley, "A new bound and algorithm for star 16QAM carrier phase estimation," IEEE Trans. Commun., vol. 51, no. 2, pp. 161-165, Feb. 2003.

[3] F. Rice, "Bounds and algorithms for carrier frequency and phase estimation," Ph.D. dissertation, Univ. South Australia, Adelaide, Australia, 2002.

[4] W. Cowley, "Phase and frequency estimation for PSK packets: Bounds and algorithms," IEEE Trans. Commun., vol. 44, no. 1, pp. 1-3, Jan. 1996.

[5] S. Kay, Fundamentals of Statistical Signal Processing: Estimation Theory. Englewood Cliffs, NJ: Prentice-Hall, 1993.

[6] J. A. Gansman, J. V. Krogmeier, and M. P. Fitz, "Single frequency estimation with nonuniform sampling," in Proc. 13th Asilomar Conf. Signals, Syst., Comput., Pacific Grove, CA, Nov. 1996, pp. 399-403. 